Review

\title{
Current Status and Prospects of Biodiesel Production from Microalgae
}

\section{Xiaodan Wu ${ }^{1,2}$, Rongsheng Ruan ${ }^{1,2,3}$, Zhenyi Du ${ }^{3}$ and Yuhuan Liu ${ }^{1,2, *}$}

1 The State Key Laboratory of Food Science and Technology, Nanchang University, Nanchang 330047, China; E-Mails: wuxiaodan0412@163.com (X.W.); ruanx001@umn.edu (R.R.)

2 The Engineering Research Center for Biomass Conversion, MOE, Nanchang University, Nanchang 330047, China

3 Center for Biorefining and Department of Bioproducts and Biosystems Engineering, University of Minnesota, St. Paul, MN 55108, USA; E-Mail:duxxx115@umn.edu

* Author to whom correspondence should be addressed; E-Mail: liuyuhuan@ncu.edu.cn; Tel.: +86-791-88333281; Fax: +86-791-88333281.

Received: 15 May 2012; in revised form: 13 July 2012 / Accepted: 20 July 2012 /

Published: 25 July 2012

\begin{abstract}
Microalgae represent a sustainable energy source because of their high biomass productivity and ability to remove air and water born pollutants. This paper reviews the current status of production and conversion of microalgae, including the advantages of microalgae biodiesel, high density cultivation of microalgae, high-lipid content microalgae selection and metabolic control, and innovative harvesting and processing technologies. The key barriers to commercial production of microalgae biodiesel and future perspective of the technologies are also discussed.
\end{abstract}

Keywords: microalgae; biodiesel; high density; high lipid content; processing; advanced technology; sustainability

\section{Introduction}

In recent years, increasing consumption of conventional energy has caused serious concerns about energy security and environmental degradation. Therefore, renewable, non-polluting biomass energy has been receiving more and more attention from both the academic community and industries. Biomass derived from photosynthesis includes a variety of organisms, such as plants, animals and 
microbes. As photosynthetic microorganisms, microalgae can use and therefore remove nitrogen, phosphorus in wastewater, sequester $\mathrm{CO}_{2}$ in the air, and synthesize lipids which can be converted into biodiesel. The declining supply of conventional fossil fuel and concern about global warming make microalgae-based biodiesel a very promising alternative. Although the potential and advantages of microalgae-based biodiesel over conventional biodiesel have been well recognized [1-3], broad commercialization of microalgae biodiesel has not yet to be realized, chiefly because of the techno-economic constraints, particularly in the areas of mass cultivation and downstream processing. The objectives of the present paper are to review recent development in microalgae production, especially in high density cultivation and downstream processing, and identify technological bottlenecks and strategies for further development.

\section{The Superiority of Microalgae Biodiesel}

As an alternative feedstock for biodiesel production, microalgae have the following advantages over conventional oil crops such as soybeans: (1) microalgae have simple structures, but high photosynthetic efficiency with a growth doubling time as short as $24 \mathrm{~h}$. Moreover, microalgae can be produced all year round. Some data in Table 1 [4] show microalgae are the only source of biodiesel that have the potential to completely displace fossil diesel. (2) The species abundance and biodiversity of microalgae over a broad spectrum of climates and geographic regions make seasonal and geographical restrictions much less of a concern compared with other lipid feedstocks. Microalgae may be cultivated on freshwater, saltwater lakes with eutrophication, oceans, marginal lands, deserts, etc. (3) Microalgae can effectively remove nutrients such as nitrogen and phosphorus, and heavy metals from wastewaters. (4) Microalgae sequester a large amount of carbons via photosynthesis, for example, the $\mathrm{CO}_{2}$ fixation efficiency of Chlorella vulgaris was up to $260 \mathrm{mg} \cdot \mathrm{L}^{-1} \cdot \mathrm{h}^{-1}$ in a membrane photobioreactor [5]. Utilization of $\mathrm{CO}_{2}$ from thermal power plants by large-scale microalgae production facilities can reduce a great deal of the greenhouse gas emissions blamed for global warming. (5) The production and use of microalgae biodiesel contribute near zero net $\mathrm{CO}_{2}$ and sulfur to the atmosphere. (6) Microalgae can produce a number of valuable products, such as proteins, polysaccharides, pigments, animal feeds, fertilizers, and so on. In short, microalgae are a largely untapped biomass resource for renewable energy production.

Table 1. Comparison of some sources of biodiesel.

\begin{tabular}{|c|c|}
\hline Crop & Oil yield $\left({\left.\mathrm{L} \cdot h a^{-1}\right)}^{2}\right.$ \\
\hline Corn & 172 \\
\hline Soybean & 446 \\
\hline Canola & 1,190 \\
\hline Jatropha & 1,892 \\
\hline Coconut & 2,689 \\
\hline Oil palm & 5,950 \\
\hline Microalgae ( $70 \%$ oil in biomass) & 136,900 \\
\hline Microalgae ( $30 \%$ oil in biomass) & 58,700 \\
\hline
\end{tabular}


However, commercialization of microalgae biomass and biofuel production is still facing significant obstacles due to high production costs and poor efficiency. In face of these challenges, researchers are undertaking profound efforts to improve microalgae biomass production and lipid accumulation and lower downstream processing costs.

\section{High Density Cultivation of Microalgae}

As simple photosynthetic organisms, microalgae can fix $\mathrm{CO}_{2}$ and synthesize organic compounds, such as lipids, proteins and carbohydrates in large amounts over short periods of time. Traditional methods of microalgae cultivation based on photoautotrophic mode have many shortcomings, among which low cell density is a major issue giving rise to low productivity, harvesting difficulty, associated high costs, and hence poor techno-economic performance. Therefore, a signficant effort towards commercializing microalgae biomass production is to develop high density cultivation processes. Two approaches are being actively researched and developed: (1) metabolic pathways control; (2) cultivation system design.

\subsection{Metabolic Pathways}

Microalgae may utilize one or more of the three major metabolic pathways depending on light and carbon conditions: photoautotrophy, heterotrophy, and mixotrophy [6]. Most microalgae are capable of photoautotrophic growth. Photoautotrophic cultivation in open ponds is a simple and low-cost way for large-scale production; however the biomass density is low because of limited light transmission, contamination by other species or bacteria, and low organic carbon concentration [7]. Some microalgae can make use of organic carbons and $\mathrm{O}_{2}$ to undergo rapid propagation through heterotrophic pathway. Heterotrophic cultivation has drawn increasing attention and it is regarded as the most practical and promising way to increase the productivity [8-10]. Currently, research on heterotrophic cultivation of microalgae is mainly focused on Chlorella. Cell densities as high as $104.9 \mathrm{~g} \cdot \mathrm{L}^{-1}$ (dry cell weight, Chlorella pyrenoidosa) have been reported [11]. Microalgae can adapt to different organic matters such as sucrose, glycerol, xylan, organic acids in slurry after acclimatization [12]. The ability of heterotrophic microalgae to utlize a wide variety of organic carbons provides an opportunity to reduce the overall cost of microalgae biodiesel production since these organic substrates can be found in the waste streams such as animal and municipal wastewaters, effluents from anaerobic digestion, food processing wastes, etc. On the basis of heterotrophic cultivation, researchers have carried out studies of mixotrophic cultivation which can greatly enhance the growth rate because it realizes the combined effects of photosynthesis and heterotrophy. After examining the biomass and lipid productivities characteristics of 14 microalgae, Park et al. [13] found that biomass and lipid productivities were boosted by mixotrophic cultivation. Andrade et al. [14] studied the effects of molasses concentration and light levels on mixotrophic growth of Spirulina platensis, and found the biomass production was stimulated by molasses, which suggested that this industrial by-product could be used as a low-cost supplement for the growth of this species. Bhatnagar et al. [15] found the mixotrophic growth of Chlamydomonas globosa, Chlorella minutissima and Scenedesmus bijuga resulted in 3-10 times more biomass production compared to that obtained under phototrophic growth conditions. The maximum lipid productivities of Phaeodactylum 
tricornutum in mixotrophic cultures with glucose, starch and acetate in medium were $0.053,0.023$ and $0.020 \mathrm{~g} \cdot \mathrm{L}^{-1} \cdot \mathrm{day}^{-1}$, which were respectively 4.6-, 2.0-, and 1.7-fold of those obtained in the corresponding photoautotrophic control cultures [16].

\subsection{Cultivation Systems}

In order to achieve large-scale biodiesel production from microalgae, a cost effective cultivation system is of great significance. The cultivation systems include open and closed styles. The former, which simulates the growth environment in natural lakes, is just open-ponds characterized by simple and low cost structure and operations, low biomass concentration, and poor system stability. The closed culture systems are photobioreactors (PBR) of different configurations including tubular, flat plate, and column photobioreactors. Compared with open pond systems, the closed systems are usually more stable because it is easier to control the process conditions and maintain monoculture and allow higher cell density, but they have higher capital and operational costs. In both open and closed microalgae culture systems, light source and light intensity are critical to the performance of phototrophic growth of microalgae. With the development of optical trapping system, light delivery and lighting technologies, which improve the distribution and absorption of light, the advent of some new photobioreactors will improve the efficiency of photosynthesis [17]. In addition, gas-liquid mass transfer efficiency is another critical factor affecting $\mathrm{CO}_{2}$ utilization and hence the phototrophic growth. Cheng et al. [18] constructed a $10 \mathrm{~L}$ photobioreactor integrated with a hollow fiber membrane module which increased the gas bubbles retention time from $2 \mathrm{~s}$ to more than $20 \mathrm{~s}$, increasing the $\mathrm{CO}_{2}$ fixation rate of Chlorella vulgaris from 80 to $260 \mathrm{mg} \cdot \mathrm{L}^{-1} \cdot \mathrm{h}^{-1}$.

\section{High Lipid Content of Microalgae}

It is easy to increase the chemical composition of microalgae by changing the environmental conditions and other factors. Many studies indicate that the composition, including lipid content, of microalgae is closely related to the environmental conditions and medium composition. Improving the lipid content in microalgae is a focus of commercial production of microalgae biomass. Current studies on high lipid content of microalgae are focused mainly on selection of microalgae species, genetic modification of microalgae, nutrient management, metabolic pathways, cultivation conditions, and so on.

\subsection{Microalgae Species and Strains}

The lipid content of microalgae varies among different species and strains (Table 2 [19,20]). The lipid content of microalgae is usually in the range of $20 \%$ to $50 \%$ (dry base), and can be as high as $80 \%$ under certain circumstances. Selecting high lipid content and fast growing microalgae is an important step in the overall success of biodiesel production from microalgae. Traditional methods of screening microalgae for high lipid content rely on time-consuming and laborious lipid extraction process which involves cell wall disruption and solvent extraction of a reasonably large amount of microalgae cells. Recently, high-throughput screening techniques employing lipophilic fluorescent dye staining (such as Nile Red [21], BODIPY 505 [22]) and fluorescence microscopy or flow cytometry 
are being developed [23]. With these new techniques, the amount of sample and preparation time are greatly reduced because the lipid content of algal cells is measured in situ without the need for extraction. Lipid content and lipid productivity are two different concepts. The former refers to lipid concentration within the microalgae cells without consideration of the overall biomass production. However the latter takes into account both the lipid concentration within cells and the biomass produced by these cells. Therefore lipid productivity is a more reasonable indicator of a strain's performance in terms of lipid production.

Table 2. Lipid content in the dry biomass of various species of microalgae.

\begin{tabular}{|c|c|}
\hline Species & Lipid content (\% dryweight) \\
\hline Anabaena cylindrica & $4-7$ \\
\hline Botyococcus braunii & $25-80$ \\
\hline Chlamydomonas reinhardtii & 21 \\
\hline Chlorella emersonii & $28-32$ \\
\hline Chlorella protothecoides & 57.9 \\
\hline Chlorella pyrenoidosa & 2 \\
\hline Chlorella vulgaris & $14-22$ \\
\hline Crypthecodinium cohnii & 20 \\
\hline Cylindrotheca sp. & $16-37$ \\
\hline Dunaliella bioculata & 8 \\
\hline Dunaliella primolecta & 23 \\
\hline Dunaliella salina & 6 \\
\hline Dunaliella tertiolecta & 35.6 \\
\hline Euglena gracilis & $14-20$ \\
\hline Hormidium sp. & 38 \\
\hline Isochrysis sp. & $25-33$ \\
\hline Monallanthus salina & $>20$ \\
\hline Nannochloris sp. & $30-50$ \\
\hline Nannochloropsis sp. & $31-68$ \\
\hline Neochloris oleoabundans & $35-54$ \\
\hline Nitzschia $\mathrm{sp}$ & $45-47$ \\
\hline Phaeodactylum tricornutum & $20-30$ \\
\hline Pleurochrysis carterae & $30-50$ \\
\hline Porphyridium cruentum & $9-14$ \\
\hline Prymnesium parvum & $22-38$ \\
\hline Scenedesmus dimorphus & $16-40$ \\
\hline Scenedesmus obliquus & $12-14$ \\
\hline Schizochytrium sp. & $50-77$ \\
\hline Spirogyra sp. & $11-21$ \\
\hline Spirulina maxima & $6-7$ \\
\hline Spirulina platensis & $4-9$ \\
\hline Synechoccus sp. & 11 \\
\hline Tetraselmis maculata & 8 \\
\hline Tetraselmis sueica & $15-23$ \\
\hline
\end{tabular}




\subsection{Genetic Modification}

The screening of strains from local habitats and elsewhere should be considered as the first step in selecting high performance strains for biodiesel production. However, other approaches including genetic manipulation may be employed to optimize the lipid and biomass productivity of promising strains obtained through the screening process. The modern biotechnologies, such as genetic engineering, cell fusion, ribosome engineering, metabolic engineering, etc. are potential techniques to develop new algae strains with rapid growth and high lipid content [24,25]. Such techniques are the key to breakthroughs in microalgae biomass energy development. In the fatty acid biosynthesis pathway, acetyl-CoA carboxylase (ACCase) is the key rate-limiting enzyme that helps the substrates acetyl-CoA enter the carbon chain of fatty acids. Therefore, it is effective to enhance the expression of ACCase to promote the lipid synthesis in microalgae. Song et al. [26] successfully constructed a vector called pRL-489-ACC to realize the shuttle expression of the gene coding acetyl-CoA carboxylase in the fatty acid synthesis pathway. Zaslavskaia et al. [27] introduced a gene encoding a glucose transporter (glutl or hupl) into Phaeodactylum tricornutumcan to allow the alga to grow on exogenous glucose in the absence of light. This represents progress of large-scale commercial production of microalgae with high lipid content by reducing limitations associated with light-dependent growth. In addition, phosphoenolpyruvate carboxylase (PEPC) is closely related to the fatty acid biosynthesis pathway because of the inhibition of PEPC activity redounding to catalyse acetyl-CoA to enter the fatty acid synthesis pathway. With successful clone of some PEPC gene in microalgae (such as Anabaena sp. PCC 7120 [28], Synechococcus vulcanus [29]) and detailed analysis of its sequence characteristics and structure, it will be possible to improve the lipid content of microalgae by regulation of PEPC expression by antisense technology [25].

\subsection{Nutrient Management}

Usually microalgae only synthesize small amounts of triacylglycerols (TAGs) under normal nutrient conditions, but can synthesize a large number of TAGs with a significant change in the fatty acid composition under stress conditions. Limiting nutrient availability such as nitrogen and phosphorus starvation during microalgae cultivation is a common method to induce lipid synthesis [30,31]. When the nitrogen is exhausted and becomes the limiting factor, microalgae will continue to absorb organic carbons, which are to be converted to lipids. The nutrient limitation also results in a gradual change in lipid composition, i.e., from free fatty acids to TAGs which are more suitable for biodiesel production [32]. Phosphorus is another important nutrient that influences algae growth and lipid accumulation. Khozin-Goldberg et al. [33] found that phosphate limitation could cause significant changes in the fatty acid and lipid composition of Monodus subterraneus. Some studies found that phosphorus deficiency led to reduced lipid content of Nannochloris atomus and Tetraselmis sp. [34]. Silicon is a necessary element for the growth of diatom. Roessler [35] found that silicon deficiency could induce lipid accumulation in Cyclotella cryptica by two distinct processes: (1) An increase in the proportion of newly assimilated carbons which are converted to lipids; (2) A slow conversion of previously assimilated carbon from non-lipid compounds to lipids. Unfortunately, higher lipid content achieved through nutrient limitation is usually at the expense of lower biomass 
productivity because nutrient deficiency limits cell growth. As mentioned above, lipid productivity, representing the combination of lipid content and biomass yield, is a more meaningful performance index to indicate the ability of lipid production of microalgae. Therefore, it is necessary to develop a nutrient management strategy which will first facilitate rapid biomass accumulation and then induce lipid accumulation in order to achieve maximum lipid productivity.

\subsection{Metabolic Pathway Management}

The lipid content of algal cells is closely related to the metabolic pathways that the algae undergo. Photoautotrophic growth is usually characterized by lower rates and lower lipid content compared with heterotrophic growth in which algae use organic matter as carbon and energy source [36,37]. Efforts have been made to control metabolic activities during cultivation in order to maximize lipid accumulation. Wu et al. [38] added organic carbon (glucose) to and reduced inorganic nitrogen in the cultivation of Chlorella protothecoides, and found that the induced heterotrophic growth resulted in $55.2 \%$ lipid content, which was about four times that in photoautotrophic growth. Xiong et al. [39] developed a photosynthesis-fermentation model with double $\mathrm{CO}_{2}$ fixation in both photosynthesis and fermentation stages which provided an efficient approach for the production of algal lipid. In this model, cultivation of $C$. protothecoides could realize $69 \%$ higher lipid yield on glucose achieved at the fermentation stage, and $61.5 \%$ less $\mathrm{CO}_{2}$ released compared with typical heterotrophic metabolism.

\subsection{Culture Conditions}

Lipid synthesis may also be induced under other stress culture conditions, such as extreme light [40], temperature [41], salinity [42], $\mathrm{pH}$ [43], and $\mathrm{CO}_{2}$ conditions [44]. Therefore, during cultivation, process conditions must be closely monitored and/or appropriately adjusted for optimal lipid productivity.

\section{Processing of Microalgae Lipid}

\subsection{Microalgae Harvesting and Dewatering}

Microalgae cells are small (typically in the range of $\Phi 2-70 \mu \mathrm{m}$ ) and the cell densities in culture broth are low (usually in the range of $0.3-5 \mathrm{~g} \cdot \mathrm{L}^{-1}$ ). Harvesting microalgae from the culture broth and dewatering them are energy intensive and therefore a major obstacle to commercial scale production and processing of microalgae. Many harvesting technologies, such as centrifugation, flocculation, filtration, gravity sedimentation, floatation, and electrophoresis techniques have been tested [45]. The choice of harvesting technique depends on, in part, the characteristics of microalgae (such as their size and density) and the target products.

\subsection{Microalgae Lipid Extraction and Refining}

Intracellular microalgae lipids can be extracted by a variety of methods, such as mechanical crushing extraction, chemical extraction, enzymatic extraction, supercritical carbon dioxide $\left(\mathrm{SCCO}_{2}\right)$ extraction [46], microwave extraction [47], etc. 
Microalgae lipids in the form of triglycerides or fatty acids can be converted to biodiesel through transesterification/(esterification for fatty acids) reactions after the extraction [48]. In order to achieve efficient reaction, the choice of catalyst is very important. The traditional liquid acid and alkali catalyst are called homogeneous catalysts because they act in the same liquid phase as the reaction mixture. Due to their simple usage and less time required for lipids conversion, the homogeneous catalysts dominate the biodiesel industry. However, the transesterification catalyzed by homogeneous catalysts needs high purity feedstock and complicated downstream processing [49], so high efficiency and low pollution catalysts such as solid acid catalysts, solid alkali catalysts, enzyme catalyst, supercritical catalyst systems and ionic liquid catalysts are receiving increasing attentions. Krohn et al. [50] studied the catalytic process using supercritical methanol and porous titania microspheres in a fixed bed reactor to catalyze the simultaneous transesterification and esterification of triglycerides and free fatty acids to biodiesel. The process was able to reach conversion efficiencies of up to $85 \%$. Patil et al. [51] reported a process involving simultaneous extraction and transesterification of wet algal biomass containing about $90 \%$ of water under supercritical methanol conditions.

\subsection{Pyrolysis of Microalgae Lipid}

Thermochemical conversion covers different processes such as direct combustion, gasification, thermochemical liquefaction, and pyrolysis. In pyrolysis, heating rate affects reaction rate which in turn affects product composition. Traditional heating methods require expensive heating mechanisms to achieve rapid temperature rise with poor process control. Microwave assisted pyrolysis (MAP) has following advantages: fine grinding of biomass is not necessary; microwave heating is mature and scalable technology which is suitable for distributed biomass conversion. Due to insufficient understanding of the mechanism of pyrolysis and the lack of effective control of the pyrolysis process, pyrolytic bio-oils are complex mixture with low calorific value, high acidity, high oxygen volume, and poor stability. However, bio-oil from pyrolysis of microalgae appears to have higher quality than those from cellulosics [52].

In recent years, the role of catalyst and minerals in biomass pyrolysis was investigated. $\mathrm{Lu}$ et al. [53] reported that $\mathrm{ZnCl}_{2}$ could catalyze fast pyrolysis of corn cob with the main products of furfural and acetic acid. Du et al. [54] used 1-butyl-3-methylimidazolium chloride and 1-butyl-3-methylimidazolium boron tetrafluoride as the catalysts in MAP of straw and sawdust.

\section{The Bottleneck of Commercial Development of Microalgae Biodiesel}

Although the potential of microalgae lipid production is tremendous, no commercial development of microalgae based fuels has been achieved so far because of lack of price competitiveness versus petroleum diesel. The bottleneck that limits the development of microalgae biomass energy is that we do not have technologies to produce large quantities of low-cost and high lipid content microalgae biomass. In order to achieve large-scale and low-cost cultivation of microalgae, the following bottleneck issues should be addressed. 


\subsection{Development of High Performance Microalgae Strains}

Breeding of high-quality microalgae with characteristics of high lipid productivity and strong adaptability is the key to realize both of high lipid content and great biomass. Excellent microalgae strains can be obtained by screening of a wide range of naturally available isolates, and the efficiency of those can be improved by selection and transformation. Considering the good results of mixotrophic cultivation, we should do some domestication making microalgae adapt to different organic matters such as sucrose, glycerol, xylan, organic acids in slurry. This will hopefully greatly reduce the cost of cultivation.

\subsection{Development of Cost-Effective Cultivation Systems}

Continuous production systems are a critical element in large scale commercial production of algal biomass. While we should continue to improve open pond operations, significant efforts should be invested in development of photobioreactors for high density cultivation of microalgae. Photobioreactors with a real time smart on-line monitoring system which can maintain optimal conditions for the growth of microalgae is very promising to realize high growth rate and cell density [55]. In order to address the issues with high capital and operational costs, innovative photobioreactor designs must be developed. Such designs may incorporate cost effective lighting techniques and renewable power combining solar energy, biogas, wind energy, waste heat.

\subsection{Wastewater Treatment and Microalgae Cultivation}

Disposal of wastewaters from human activities and animal production is both an environmental and a financial issue. These nitrogen and phosphorus rich wastewaters have been proven suitable for microalgae growth. The feasibility of growing microalgae to remove inorganic nitrogen, phosphorus, metal elements and other pollutants in the wastewaters has long been recognized [56,57]. Therefore, a wastewater based microalgae production process has the due benefits of wastewater treatment and production of algal biomass with minimum external input of fresh water and nutrients. It has been argued that microalgae biodiesel production in conjunction with wastewater treatment is the area with the most plausible commercial potential in the short term [58,59]. Several applications in wastewater treatment have been reported in the literature. Chinnasamy et al. [60] found that both fresh water and marine algae showed good growth in carpet industry effluents and municipal sewage. Kim et al. [61] added fermented swine urine (3\%) (v/v) to the medium to culture Scenedesmus sp., and received the growth rate (3-fold), dry weight (2.6-fold). Wang et al. [62] investigated the effectiveness of using digested dairy manure as a nutrient supplement for cultivation of oil-rich green microalgae Chlorella sp, and found that the total fatty acid content of dry weight was increased from $9.00 \%$ to $13.7 \%$, along with removal of ammonia, total nitrogen, total phosphorus, and COD by $100 \%$, $75.7 \%-82.5 \%, 62.5 \%-74.7 \%$, and $27.4 \%-38.4 \%$, respectively. Li et al. [63] cultivated Scenedesmus sp. LX1 in secondary effluent, achieving high biomass yield $\left(0.11 \mathrm{~g} \cdot \mathrm{L}^{-1}\right.$, dry weight $)$ and lipid content (31\%-33\%, dry weight). 


\section{4. $\mathrm{CO}_{2}$ Fixation and Microalgae Cultivation}

To significantly improve photoautotrophic cultivation, new microalgae strains and process conditions must be developed to enable fast growth and high lipid accumulation at high $\mathrm{CO}_{2}$ levels $[64,65]$. If $\mathrm{CO}_{2}$ is from flue gas, algae strains' high tolerance to $\mathrm{SO}_{x}, \mathrm{NO}_{x}$, and high temperature is desirable [66]. Morais et al. [67-69] isolated several microalgae from the waste treatment ponds of a coal fired thermoelectric power plant, and investigated their growth characteristics when exposed to different concentrations of $\mathrm{CO}_{2}$. When cultivated with $6 \%$ and $12 \% \mathrm{CO}_{2}$, Chlorella kessleri showed a maximum biomass productivity at $6 \% \mathrm{CO}_{2}$ while Scenedesmus obliquus showed a maximum biomass productivity at $12 \% \mathrm{CO}_{2}$. They also found that Spirulina sp., Scenedesmus obliquus, and Chlorella vulgaris grew well when the culture medium contained up to $18 \% \mathrm{CO}_{2}$, and Spirulina sp. exhibited the highest rate among them. Chang et al. [70] found that some strains of Chlorella could grow in an atmosphere containing $\mathrm{CO}_{2}$ up to $40 \%$. Base on these studies, we can try to use some means to promote microalgae growth stimulated by $\mathrm{CO}_{2}$ addition [71]. Such as pump $\mathrm{CO}_{2}$ emission from power plants, industrial processes, or soluble carbonate through a sparger system of orifices evenly distributed over the bottom of the ponds when cultivating microalgae in large open-ponds.

\subsection{Development of Immobilization Technique}

The algal cells can be gathered into a corporation or fixed to the carrier by immobilization technology. Immobilized algae have numerous benefits as follows: high cell density, excellent ability to resist poison, high removal efficiency, good stability, easy product separation, etc. At present, immobilization carriers include mainly natural macromolecular materials (such as agar, sodium alginate, etc.) and synthetic polymeric gels (such as polyvinyl alcohol, polyacrylamide, etc.). The natural polymers have no toxicity and good mass transfer property, but their intensity is low. However, the synthetic organic polymer gels have high strength, but poor mass transfer property. Therefore, new immobilization carriers are expected to facilitate the development of algae immobilization technology.

High rate algal pond (HRAP) can achieve efficient removal of organic matter, heavy metals, nitrogen, phosphorus and other nutrients in wastewater by algae-bacteria symbiosis system. In the system, the microalgae provide oxygen and nutrition for bacteria; meanwhile bacteria provide useful growth-promoting substances for microalgae. Our team has developed a new type of microalgae immobilization carrier using the fungi [72]. The key of the technology is that fungi mediate pelletization of microalgae. After adding a certain number of fungal spores to the microalgae culture, algae-fungi symbiotic spheres formed accompanied by spore germination. The immobilized microalgae allow efficient recycling of livestock wastewater for algae cultivation and are easy to be harvested. In addition, the fungi can be converted to animal feed.

\subsection{Extension of Microalgae Chain}

In addition to lipids, microalgae can produce some value-added bioactive substances, such as polysaccharides, proteins and pigments. Therefore, microalgae have good prospects in the food, feed, pharmaceutical, and cosmetic industries. If a biorefinery approach that converts microalgae to wide 
range of products including biodiesel and value-added products, it will significantly improve the economic outlook of microalgae based fuels production.

\section{Conclusions}

Microalgae are a sustainable energy resource with great potential for $\mathrm{CO}_{2}$ fixation and wastewater purification. This review discusses current status and anticipated future developments in the microalgae to biodiesel approach. For biodiesel production to have a significant impact on renewable fuels standards, technologies must be developed to enable large scale algae biomass production. The strategies for both high biomass and lipid productivities are discussed. Further efforts on microalgae biodiesel production should focus on reducing costs in large-scale algal biomass production systems. Combining microalgae mixotrophic cultivation with sequestration of $\mathrm{CO}_{2}$ from flue gas and wastewater treatment, and taking the biorefinery approach to algal biomass conversion will improve the environmental and economic viability.

\section{Acknowledgments}

The authors are grateful for financial support of the Natural Science Foundation of China (30960304), of the Key Programme for Bioenergy Industrialization of Jiangxi Provincial Department of Science and Technology (2007BN12100), of the International Science and Technology Cooperation Programme of China (2010DFB63750), of the International Science and Technology Cooperation Programme of Jiangxi Provin-cial Department of Science and Technology (2010EHB03200), of the research programe of State Key Laboratory of Food Science and Technology, Nanchang university (SKLF-TS-201111 and SKLF-TS-200814), of 948 Programe of State Forestry Bureau (2010-4-09), and National High-tech R\&D Program of China (2012AA101800-03, 2012AA021704, and 2012AA021205).

\section{References}

1. Khan, S.A.; Rashmi; Hussain, M.Z.; Prasad, S.; Banerjee, U.C. Prospects of biodiesel production from microalgae in India. Renew. Sustain. Energy Rev. 2009, 13, 2361-2372.

2. Lim, S.; Teong, L.K. Recent trends, opportunities and challenges of biodiesel in Malaysia: An overview. Renew. Sustain. Energy Rev. 2010, 14, 938-954.

3. Stephens, E.; Ross, I.L.; King, Z.; Mussgnug, J.H.; Kruse, O.; Posten, C.; Borowitzka, M.; Hankamer, B. An economic and technical evaluation of microalgal biofuels. Nature Biotechnol. 2010, 28, 126-128.

4. Chisti, Y. Biodiesel from microalgae. Biotechnol. Adv. 2007, 25, 294-306.

5. Cheng, L.H.; Zhang, L.; Chen, H.L.; Gao, C.J. Carbon dioxide removal from air by microalgae cultured in a membrane-photobioreactor. Sep. Purif. Technol. 2006, 50, 324-329.

6. Chojnacka, K.; Noworyta, A. Evaluation of Spirulina sp. growth in photoautotrophic, heterotrophic and mixotrophic cultures. Enzyme Microb. Technol. 2004, 34, 461-465. 
7. Greenwell, H.C.; Laurens, L.M.L.; Shields, R.J.; Lovitt, R.W.; Flynn, K.J. Placing microalgae on the biofuels priority list: A review of the technological challenges. J. R. Soc. Interface 2010, 7 , 703-726.

8. Chen, F. High cell density culture of microalgae in heterotrophic growth. Trends Biotechnol. 1996, $14,421-426$.

9. Li, X.F.; Xu, H.; Wu, Q.Y. Large-scale biodiesel production from microalga Chlorella protothecoides through heterotrophic cultivation in bioreactors. Biotechnol. Bioeng. 2007, 98, 764-771.

10. Doucha, J.; Lívansky, K. Production of high-density Chlorella culture grown in fermenters. J. Appl. Phycol. 2012, 24, 35-43.

11. Wu, Z.Y.; Shi, X.M. Optimization for high-density cultivation of heterotrophic Chlorella based on a hybrid neural network model. Lett. Appl. Microbiol. 2006, 44, 13-18.

12. Heredia-Arroyo, T.; Wei, W.; Ruan, R.; Hu, B. Mixotrophic cultivation of Chlorella vulgaris and its potential application for the oil accumulation from non-sugar materials. Biomass Bioenergy 2011, 35, 2245-2253.

13. Park, K.C.; Whitney, C.; McNichol, J.C.; Dickinson, K.E.; MacQuarrie, S.; Skrupski, B.P.; Zou, J.; Wilson, K.E.; O’Leary, S.J.B.; McGinn, P.J. Mixotrophic and photoautotrophic cultivation of 14 microalgae isolates from Saskatchewan, Canada: Potential applications for wastewater remediation for biofuel production. J. Appl. Phycol. 2012, 24, 339-348.

14. Andrade, M.R.; Costa, J.A.V. Mixotrophic cultivation of microalga Spirulina platensis using molasses as organic substrate. Aquaculture 2007, 264, 130-134.

15. Bhatnagar, A.; Chinnasamy, S.; Singh, M.; Das, K.C. Renewable biomass production by mixotrophic algae in the presence of various carbon sources and wastewaters. Appl. Energy 2011, $88,3425-3431$.

16. Wang, H.Y.; Fu, R.; Pei, G.F. A study on lipid production of the mixotrophic microalgae Phaeodactylum tricornutum on various carbon sources. Afr. J. Microbiol. Res. 2012, 6, 1041-1047.

17. Masojidek, J.; Papacek, S.; Sergejevova, M.; Jirka, V.; Cerveny, J.; Kunc, J.; Korecko, J.; Verbovikova, O.; Kopecky, J.; Stys, D. A closed solar photobioreactor for cultivation of microalgae under supra-high irradiance: Basic design and performance. J. Appl. Phycol. 2003, 15, 239-248.

18. Cheng, L.H.; Zhang, L.; Chen, H.L.; Cao, C.J. Carbon dioxide removal from air by microalgae cultured in a membrane-photobioreactor. Sep. Purif. Technol. 2006, 50, 324-329.

19. Singh, A.; Nigam, P.S.; Murphy, J.D. Renewable fuels from algae: An answer to debatable land based fuels. Bioresour. Technol. 2011, 102, 10-16.

20. Singh, A.; Nigam, P.S.; Murphy, J.D. Mechanism and challenges in commercialisation of algal biofuels. Bioresour. Technol. 2011, 102, 26-34.

21. Elsey, D.; Jameson, D.; Raleigh, B.; Cooney, M.J. Fluorescent measurement of microalgal neutral lipids. J. Microbiol. Methods 2007, 68, 639-642.

22. Cooper, M.S.; Hardin, W.R.; Petersen, T.W.; Cattolico, R.A. Visualizing "green oil" in live algal cells. J. Biosci. Bioeng. 2010, 109, 198-201. 
23. Mutanda, T.; Ramesh, D.; Karthikeyan, S.; Kumari, S.; Anandraj, A.; Bux, F. Bioprospecting for hyper-lipid producing microalgal strains for sustainable biofuel production. Bioresour. Technol. 2011, 102, 57-70.

24. Rosenberg, J.N.; Oyler, G.A.; Wilkinson, L.; Betenbaugh, M.J. A green light for engineered algae: Redirecting metabolism to fuel a biotechnology revolution. Curr. Opin. Biotechnol. 2008, 19, 430-436.

25. Song, D.H.; Fu, J.J.; Shi, D.J. Exploitation of oil-bearing microalgae for biodiesel. Chin. J. Biotechnol. 2008, 24, 341-348.

26. Song, D.H.; Hou, L.J.; Song, X.F.; Shi, D.J. Cloning of the acetyl coenzyme A carboxylase gene of Cyanophyta in fatty acid biosynthesis pathway and the construction of the shuttle expression vector. In Proceedings of the 14th National Algae Symposium, Hohhot, China, 12-15 August 2007.

27. Zaslavskaia, L.A.; Lippmeler, J.C.; Shih, C.; Ehrhardt, D.; Grossman, A.R.; Apt, K.E. Trophic conversion of an obligate photoautotrophic organism through metabolic engineering. Science 2001, 292, 2073-2075.

28. Luinenburg, I.; Coleman, J.R. Identification, characterization and sequence analysis of the gene encoding phosphoenolpyruvate carboxylase in Anabaena sp. PCC 7120. J. Gen. Microbiol. 1992, 138, 685-691.

29. Chen, L.M.; Omiya, T.; Hata, S.; Izui, K. Molecular characterization of a phosphoenolpyruvate carboxylase from a thermophilic cyanobacterium, Synechococcus vulcanus with unusual allosteric properties. Plant Cell Physiol. 2002, 43, 159-169.

30. Zhila, N.O.; Kalacheva, G.S.; Volova, T.G. Effect of nitrogen limitation on the growth and lipid composition of the green alga Botryococcus braunii Kütz IPPAS H-252 Russian. J. Plant Physiol. 2005, 52, 311-319.

31. Weldy, C.S.; Huesemann, M.H. Lipid production by Dunaliella salina in batch culture: Effects of nitrogen limitation and light intensity. J. Undergrad. Res. 2007, 7, 115-122.

32. Widjaja, A.; Chien, C.C.; Ju, Y.H. Study of increasing lipid production from fresh water microalgae Chlorella vulgaris. J. Taiwan Inst. Chem. Eng. 2009, 40, 13-20.

33. Khozin-Goldberg, I.; Cohen, Z. The effect of phosphate starvation on the lipid and fatty acid composition of the fresh water eustigmatophyte Monodus subterraneus. Phytochemistry 2006, 67, 696-701.

34. Reitan, K.I.; Rainuzzo, J.R.; Olsen, Y. Effect of nutrient limitation on fatty acid and lipid content of marine microalgae. J. Phycol. 1994, 30, 972-979.

35. Roessler, P.G. Effects of silicon dificiency on lipid composition metabolism in the diatom Cyclotella cryptica. J. Phycol. 1988, 24, 394-400.

36. Miao, X.L.; Wu, Q.Y. High yield bio-oil production from fast pyrolysis by metabolic controlling of Chlorella protothecoides. J. Biotechnol. 2004, 110, 85-93.

37. Wei, D.; Liu, L.J. Optimization of culture medium for heterotrophic Chlorella Protothecoides producing total fatty acids. Chem. Bioeng. 2008, 25, 35-40.

38. Xu, H.; Miao, X.L.; Wu, Q.Y. High quality biodiesel production from a microalga Chlorella protothecoides by heterotrophic growth in fermenters. J. Biotechnol. 2006, 126, 499-507. 
39. Xiong, W.; Gao, C.F.; Yan, D.; Wu, C.; Wu, Q. Double $\mathrm{CO}_{2}$ fixation in photosynthesis-fermentation model enhances algal lipid synthesis for biodiesel production. Bioresour. Technol. 2010, 101, 2287-2293.

40. Guedes, A.C.; Meireles, L.A.; Amaro, H.M.; Malcata, F.X. Changes in lipid class and fatty acid composition of cultures of Pavlova lutheri, in response to light intensity. J. Am. Oil Chem. Soc. 2010, 87, 791-801.

41. Renaud, S.M.; Thinh, L.V.; Lambrinidis, G.; Parry, D.L. Effect of temperature on growth, chemical composition and fatty acid composition of tropical Australian microalgae grown in batch cultures. Aquaculture 2002, 211, 195-214.

42. Kotlova, E.R.; Shadrin, N.V. The role of membrane lipids in adaptation of Cladophora (Chlorophyta) to living in shallow lakes with different salinity. Botanicheskii Zhurnal 2003, 88, $38-45$.

43. Guckert, J.B.; Cooksey, K.E. Triglyceride accumulation and fatty acid profile changes in Chlorella (Chlorophyta) during high pH-induced cell cycle inhibition. J. Phycol. 1990, 26, 72-79.

44. Chiu, S.Y.; Kao, C.Y.; Tsai, M.T.; Ong, S.C.; Chen, C.H.; Lin, C.S. Lipid accumulation and $\mathrm{CO}_{2}$ utilization of Nanochloropsis oculata in response to $\mathrm{CO}_{2}$ aeration. Bioresour. Technol. 2009, 100, 833-838.

45. Uduman, N.; Qi, Y.; Danquah, M.K.; Forde, G.M.; Hoadley, A. Dewatering of microalgal cultures: A major bottleneck to algae-based fuels. J. Renew. Sustain. Energy 2010, 2, 012701.

46. Halim, R.; Gladman, B.; Danquah, M.K.; Webley, P.A. Oil extraction from microalgae for biodiesel production. Bioresour. Technol. 2011, 102, 178-185.

47. Koberg, M.; Cohen, M.; Ben-Amotz, A.; Gedanken, A. Bio-diesel production directly from the microalgae biomass of Nannochloropsis by microwave and ultrasound radiation. Bioresour. Technol. 2011, 102, 4265-4269.

48. Johnson, M.B.; Wen, Z. Production of biodiesel fuel from the microalga Schizochytrium limacinum by direct transesterification of algal biomass. Energy Fuels 2009, 23, 5179-5183.

49. Borges, M.E.; Diaz, L. Recent developments on heterogeneous catalysts for biodiesel production by oil esterification and transesterification reactions: A review. Renew. Sustain. Energy Rev. 2012, 16, 2839-2849.

50. Krohn, B.J.; McNeff, C.V.; Yan, B.; Nowlan, D. Production of algae-based biodiesel using the continuous catalytic Mcgyan ${ }^{\circledR}$ process. Bioresour. Technol. 2011, 102, 94-100.

51. Patil, P.D.; Gude, V.G.; Mannarswamy, A.; Deng, S.G.; Cooke, P.; Munson-McGee, S.; Rhodes, I.; Lammers, P.; Nirmalakhandan, N. Optimization of direct conversion of wet algae to biodiesel under supercritical methanol conditions. Bioresour. Technol. 2011, 102, 118-122.

52. Du, Z.Y.; Li, Y.C.; Wang, X.Q.; Wan, Y.Q.; Chen, Q.; Wang, C.G.; Lin, X.Y.; Liu, Y.H.; Chen, P.; Ruan, R. Microwave-assisted pyrolysis of microalgae for biofuel production. Bioresour. Technol. 2011, 102, 4890-4896.

53. Lu, Q.; Wang, Z.; Dong, C.Q.; Zhang, Z.F.; Zhang, Y.; Yang, Y.P.; Zhu, X.F. Selective fast pyrolysis of biomass impregnated with $\mathrm{ZnCl}_{2}$ : Furfural production together with acetic acid and activated carbon as by-products. J. Anal. Appl. Pyrolysis 2011, 1, 273-279.

54. Du, J.; Liu, P.; Liu, Z.H.; Sun, D.G.; Tao, C.Y. Fast pyrolysis of biomass for bio-oil with ionic liquid and microwave irradiation. J. Fuel Chem. Technol. 2010, 5, 554-559. 
55. Meireles, L.A.; Azevedo, J.L.; Cunha, J.P.; Malcata, F.X. On-line determination of biomass in a microalga bioreactor using a novel computerized flow injection analysis system. Biotechnol. Prog. 2002, 18, 1387-1391.

56. Aslan, S.; Kapdan, I. Batch kinetics of nitrogen and phosphorus removal from synthetic wastewater by algae. Ecol. Eng. 2006, 28, 64-70.

57. Shi, J.; Podola, B.; Melkonian, M. Removal of nitrogen and phosphorus from wastewater using microalgae immobilized on twin layers: An experimental study. J. Appl. Phycol. 2007, 19, 417-423.

58. Mulbry, W.; Kondrad, S.; Buyer, J. Treatment of dairy and swine manure effluents using freshwater algae: Fatty acid content and composition of algal biomass at different manure loading rates. J. Appl. Phycol. 2008, 20, 1079-1085.

59. Kong, Q.X.; Li, L.; Martinez, B.; Chen, P.; Ruan, R. Culture of microalgae Chlamydomonas reinhardtii in wastewater for biomass feedstock production. Appl. Biochem. Biotechnol. 2010, 160, 9-18.

60. Chinnasamy, S.; Bhatnagar, A.; Hunt, R.W.; Das, K.C. Microalgae cultivation in a wastewater dominated by carpet mill effluents for biofuel applications. Bioresour. Technol. 2010, 101, 3097-3105.

61. Kim, M.K.; Park, J.W.; Park, C.S.; Kim, S.J.; Jeune, K.H.; Chang, M.U.; Acreman, J. Enhanced production of Scenedesmus spp. (green microalgae) using a new medium containing fermented swine wastewater. Bioresour. Technol. 2007, 98, 2220-2228.

62. Wang, L.; Li, Y.C.; Chen, P.; Min, M.; Chen, Y.F.; Zhu, J.; Ruan, R. Anaerobic digested dairy manure as a nutrient supplement for cultivation of oil-rich green microalgae Chlorella sp. Bioresour. Technol. 2010, 101, 2623-2628.

63. Li, X.; Hu, H.Y; Yang, J. Lipid accumulation and nutrient removal properties of a newly isolated freshwater microalgae, Scenedesmus sp. LX1, growing in secondary effluent. New Biotechnol. 2010, 27, 59-63.

64. Yoo, C.; Jun, S.Y.; Lee, J.Y.; Ahn, C.Y.; Oh, H.M. Selection of microalgae for lipid production under high levels carbon dioxide. Bioresour. Technol. 2010, 101, 571-574.

65. Jiang, L.L.; Luo, S.J.; Fan, X.L.; Yang, Z.M.; Guo, R.B. Biomass and lipid production of marine microalgae using municipal wastewater and high concentration of $\mathrm{CO}_{2}$. Appl. Energy 2011, 88, $3336-3341$.

66. Lee, J.N.; Lee, J.S.; Shin, C.S.; Park, S.C.; Kim, S.W. Methods to enhance tolerances of Chlorella KR-1 to toxic compounds in flue gas. Appl. Biochem. Biotechnol. 2000, 84-86, 329-342.

67. Morais, M.G.; Costa, J.A.V. Isolation and selection of microalgae from coal fired thermoelectric power plant for biofixation of carbon dioxide. Energy Convers. Manag. 2007, 48, 2169-2173.

68. Morais, M.G.; Costa, J.A.V. Carbon dioxide fixation by Chlorella kessleri, C. vulgaris, Scenedesmus obliquus and Spirulina sp. cultivated in flasks and vertical tubular photobioreactors. Biotechnol. Lett. 2007, 29, 1349-1352.

69. Morais, M.G.; Costa, J.A.V. Biofixation of carbon dioxide by Spirulina sp. and Scenedesmus obliquus cultivated in a three-stage serial tubular photobioreactor. J. Biotechnol. 2007, 129, 439-445. 
70. Chang, E.H.; Yang, S.S. Some characteristics of microalgae isolated in Taiwan for biofixation of carbon dioxide. Bot. Bull. Acad. Sini. 2003, 44, 43-52.

71. Park, J.B.K.; Craggs, R.J. Wastewater treatment and algal production in high rate algal ponds with carbon dioxide addition. Water Sci. Technol. 2010, 61, 633-639.

72. Zhou, W.G.; Cheng, Y.L.; Li, Y.; Wan, Y.Q.; Liu, Y.H.; Lin, X.Y.; Ruan, R. Novel fungal pelletization assisted technology for algae harvesting and wastewater treatment. Appl. Biochem. Biotechnol. 2012, 167, 214-228.

(C) 2012 by the authors; licensee MDPI, Basel, Switzerland. This article is an open access article distributed under the terms and conditions of the Creative Commons Attribution license (http://creativecommons.org/licenses/by/3.0/). 